\title{
Infrared absorption band and vibronic structure of the nitrogen-vacancy center in diamond
}

\author{
P. Kehayias, ${ }^{1, *}$ M. W. Doherty, ${ }^{2}$ D. English, ${ }^{1}$ R. Fischer, ${ }^{3}$ A. Jarmola, ${ }^{1}$ K. Jensen, ${ }^{1}$ N. Leefer,,${ }^{1}$ P. Hemmer, ${ }^{4}$ \\ N. B. Manson, ${ }^{2}$ and D. Budker ${ }^{1,5, \dagger}$ \\ ${ }^{1}$ Department of Physics, University of California, Berkeley, California 94720-7300, USA \\ ${ }^{2}$ Laser Physics Centre, RSPE, Australian National University, Canberra, ACT 0200, Australia \\ ${ }^{3}$ Department of Physics, Technion - Israel Institute of Technology, Haifa 32000, Israel \\ ${ }^{4}$ Department of Electrical and Computer Engineering, Texas A\&M University, College Station, Texas 77843, USA \\ ${ }^{5}$ Nuclear Science Division, Lawrence Berkeley National Laboratory, Berkeley, California 94720, USA
}

(Received 26 January 2013; published 16 October 2013)

\begin{abstract}
Negatively charged nitrogen-vacancy $\left(\mathrm{NV}^{-}\right)$color centers in diamond have generated much interest for use in quantum technology. Despite the progress made in developing their applications, many questions about the basic properties of $\mathrm{NV}^{-}$centers remain unresolved. Understanding these properties can validate theoretical models of $\mathrm{NV}^{-}$, improve their use in applications, and support their development into competitive quantum devices. In particular, knowledge of the phonon modes of the ${ }^{1} A_{1}$ electronic state is key for understanding the optical pumping process. Using pump-probe spectroscopy, we measured the phonon sideband of the ${ }^{1} E \rightarrow{ }^{1} A_{1}$ electronic transition in the $\mathrm{NV}^{-}$center. From this we calculated the ${ }^{1} E \rightarrow{ }^{1} A_{1}$ one-phonon absorption spectrum and found it to differ from that of the ${ }^{3} E \rightarrow{ }^{3} A_{2}$ transition, a result which is not anticipated by previous group-theoretical models of the $\mathrm{NV}^{-}$electronic states. We identified a high-energy $169-\mathrm{meV}$ localized phonon mode of the ${ }^{1} A_{1}$ level.
\end{abstract}

DOI: 10.1103/PhysRevB.88.165202

PACS number(s): 78.40.Ha, 61.72.jn, 63.20.kp, 63.20.Pw

\section{INTRODUCTION}

The nitrogen-vacancy (NV) center in diamond [Fig. 1(a)] is a color center consisting of a substitutional nitrogen atom in the diamond crystal lattice adjacent to a missing carbon atom (a vacancy). NV centers have $C_{3 v}$ point-group symmetry and have discrete electronic energy states between the diamond valence and conduction bands. The negatively charged $\mathrm{NV}^{-}$center can be optically spin polarized and read out, and it has a long ground-state transverse spinrelaxation time at room temperature. ${ }^{1,2}$ These properties make $\mathrm{NV}^{-}$centers useful in a variety of applications including electric and magnetic field sensing, ${ }^{3-6}$ rotation sensing, ${ }^{7-9}$ quantum computing, ${ }^{10,11}$ quantum cryptography, ${ }^{12,13}$ and subdiffraction-limited imaging. ${ }^{14-16}$ Despite the progress made on developing these applications, the complete $\mathrm{NV}^{-}$energy-level structure and vibronic structure are unknown.

Figure 1(b) shows a simplified $\mathrm{NV}^{-}$energy-level diagram as confirmed by experiment. The triplet-triplet $\left({ }^{3} A_{2} \leftrightarrow{ }^{3} E\right)$ and singlet-singlet $\left({ }^{1} E \leftrightarrow{ }^{1} A_{1}\right)$ energy differences are known to be $1.945 \mathrm{eV}(637 \mathrm{~nm})$ and $1.190 \mathrm{eV}(1042 \mathrm{~nm})$, respectively. ${ }^{17-20}$ However, where these energy states lie with respect to the diamond valence and conduction bands is only known indirectly, as are the triplet-singlet $\left({ }^{3} A_{2} \leftrightarrow{ }^{1} A_{1}\right.$ and ${ }^{1} E \leftrightarrow$ ${ }^{3} E$ ) energy differences. ${ }^{21,22}$ Theoretical calculations predict the existence of additional energy states $\left({ }^{1} E^{\prime}\right.$ and $\left.{ }^{1} A_{1}^{\prime}\right)$, but disagree on their energies (see Refs. 23-27 and references therein). Prior experiments and ab initio calculations studied the phonon sidebands (PSBs) for the ${ }^{3} A_{2} \rightarrow{ }^{3} E$ and ${ }^{3} E \rightarrow{ }^{3} A_{2}$ transitions. ${ }^{17,28-31}$ The ${ }^{1} E \rightarrow{ }^{1} A_{1}$ and ${ }^{1} A_{1} \rightarrow{ }^{1} E$ PSBs have not been studied theoretically, and only the ${ }^{1} A_{1} \rightarrow{ }^{1} E$ transition had been measured prior to this work. ${ }^{18-20}$

A more complete experimental picture of $\mathrm{NV}^{-}$properties can provide insight for applications and validate theoretical models of $\mathrm{NV}^{-}$attributes. The 1042-nm infrared ${ }^{1} E \rightarrow{ }^{1} A_{1}$ zero-phonon line (ZPL) has been used in an absorption-based magnetometer, ${ }^{32}$ but using the ${ }^{1} E \rightarrow{ }^{1} A_{1}$ PSB instead may be more sensitive depending on the PSB structure and cross section. In addition, most $\mathrm{NV}$ experiments take advantage of an optical pumping mechanism (which involves the ${ }^{1} A_{1}$ excited vibrational states) that drives electrons to the ${ }^{3} A_{2}$ $m_{s}=0$ state. Therefore, knowledge of the ${ }^{1} E \rightarrow{ }^{1} A_{1}$ PSB could improve infrared magnetometry and optical pumping schemes. Moreover, as the $\mathrm{NV}^{-}$center develops into a mature quantum system, it is important to know the properties of the singlet states to inspire confidence that we understand this system.

We attempt to fill the gaps in the knowledge of $\mathrm{NV}^{-}$ properties by measuring the ${ }^{1} E \rightarrow{ }^{1} A_{1}$ PSB and searching for previously unobserved transitions. Finding the ${ }^{1} E \rightarrow{ }^{1} E^{\prime} \mathrm{ZPL}$ would resolve the disagreement on the predicted ${ }^{1} E^{\prime}$ energy. The ${ }^{1} E \rightarrow{ }^{1} A_{1}$ PSB yields information about the ${ }^{1} A_{1}$ phonon modes, which are also of interest. The spin-orbit interaction mixes the ${ }^{3} E$ and ${ }^{1} A_{1}$ states, resulting in triplet-singlet intersystem crossing (ISC). This enables spin-dependent nonradiative decay from the nominally ${ }^{3} E$ state to the nominally ${ }^{1} A_{1}$ state. The ISC rate is comparable to the ${ }^{3} E \rightarrow{ }^{3} A_{2}$ spontaneous decay rate ${ }^{33,34}$ and is an important factor in the optical pumping process. Measuring the ${ }^{1} A_{1}$ phonon modes could allow the optical pumping mechanism to be modeled more accurately and provide insight on $\mathrm{NV}^{-}$spin polarization and readout. Furthermore, the accepted group-theoretical model of $\mathrm{NV}^{-}$predicts ${ }^{3} A_{2}$ and ${ }^{1} A_{1}$ to have the same electronic configuration, meaning they should have the same phonon modes. A comparison between the ${ }^{3} E \rightarrow{ }^{3} A_{2}$ and ${ }^{1} E \rightarrow{ }^{1} A_{1}$ PSBs should be sensitive to differences between the ${ }^{3} A_{2}$ and ${ }^{1} A_{1}$ configurations.

In this work, we present measurements of the ${ }^{1} E \rightarrow{ }^{1} A_{1}$ ZPL and PSB. We describe the PSB absorption features, including a high-energy $(169 \mathrm{meV})$ localized phonon mode that lies outside the diamond lattice phonon density of states. 
(a)

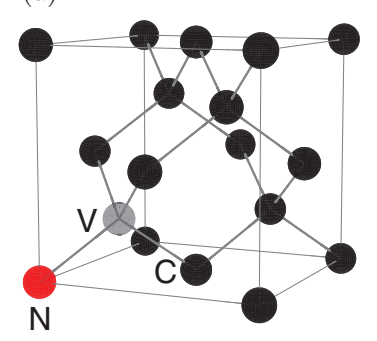

(c)
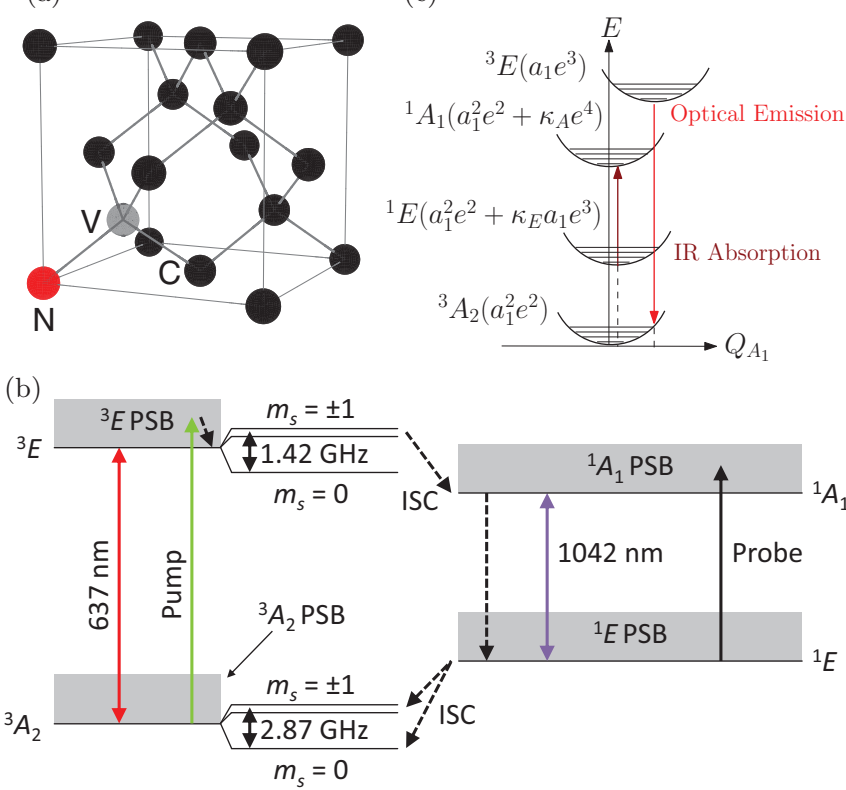

FIG. 1. (Color online) (a) The diamond lattice structure, containing an $\mathrm{NV}$ center. (b) $\mathrm{The} \mathrm{NV}^{-}$energy-level diagram and our pump-probe spectroscopy scheme. The states are labeled by their $C_{3 v}$ representations and electron-spin multiplicities. Solid arrows are optical and microwave transitions, and dashed arrows are nonradiative transitions. The label "ISC" indicates intersystem crossing, which occurs primarily for the ${ }^{3} E m_{s}= \pm 1$ states and is responsible for optical pumping. (c) A configuration coordinate diagram for $A_{1}$ phonon modes showing the harmonic nuclear potential wells and phonon energy levels. The configuration for each electronic state is denoted in parentheses, and $Q_{A_{1}}$ is the normal nuclear coordinate. With no electronic Coulomb repulsion, the ${ }^{3} A_{2},{ }^{1} E$, and ${ }^{1} A_{1}$ levels are of the $a_{1}^{2} e^{2}$ configuration and the ${ }^{3} E$ level is of the $a_{1} e^{3}$ configuration. With Coulomb repulsion included to first order, the ${ }^{1} E$ and ${ }^{1} A_{1}$ levels couple with the ${ }^{1} E^{\prime}$ (configuration $a_{1} e^{3}$ ) and ${ }^{1} A_{1}^{\prime}$ (configuration $e^{4}$ ) levels, respectively. This coupling is denoted by the parameters $\kappa_{E}$ and $\kappa_{A}$.

Comparing the ${ }^{1} E \rightarrow{ }^{1} A_{1}$ and the ${ }^{3} E \rightarrow{ }^{3} A_{2}$ phonon modes, we find that the ${ }^{1} A_{1}$ phonon modes are shifted to higher energies, meaning that proper descriptions of the ${ }^{1} A_{1}$ and ${ }^{3} A_{2}$ states require corrections to their electronic configurations.

\section{EXPERIMENT AND RESULTS}

In our experiment, we populated the metastable ${ }^{1} E$ state using pump-laser light and measured transmission of probelaser light through a diamond sample containing an ensemble of $\mathrm{NV}^{-}$centers [Figs. 1(b) and 2]. We determined the probe transmission through the diamond with and without $\mathrm{NV}^{-}$ centers in the ${ }^{1} E$ state. A 532-nm frequency-doubled Nd: $\mathrm{YVO}_{4}$ pump laser beam and a 5-mW supercontinuum probe laser beam (wavelength range $450-1800 \mathrm{~nm}$ ) were combined on a dichroic beamsplitter and focused with a $40 \times$ microscope objective ( 0.6 numerical aperture) onto a cryogenically cooled diamond sample. The transmitted light was collimated and detected with a spectrometer with $\sim 1$-nm resolution. A chopper wheel modulated the pump light and a computer collected a transmission spectrum each time the pump light was blocked

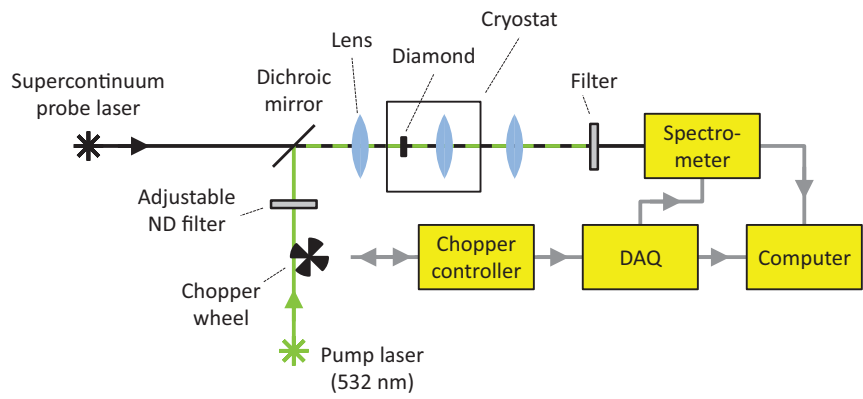

FIG. 2. (Color online) The experimental apparatus. The data acquisition device (DAQ) monitors the chopper wheel state and triggers a spectrum acquisition when the pump is blocked or unblocked. The computer collects "pump blocked" and "pump unblocked" transmission spectra.

and unblocked. Absorption from ${ }^{1} E$ appeared as a difference between the "pump blocked" and "pump unblocked" supercontinuum transmitted intensities. In another experiment, we used 912- and 1042-nm continuous-wave (cw) lasers as probe sources and replaced the spectrometer with a photodiode. ${ }^{35}$

Figure 3(a) shows the ${ }^{1} E \rightarrow{ }^{1} A_{1}$ ZPL and PSB supercontinuum absorption spectrum taken at $10 \mathrm{~K}$ with the sample "B8," a synthetic type Ib high-pressure high-temperature (HPHT) diamond with $\sim 10$ ppm $\mathrm{NV}^{-}$concentration. The PSB includes narrow absorption lines at 811 and $912 \mathrm{~nm}$ and broad absorption features at 872, 922, 931, and $983 \mathrm{~nm}$. In the figure we observe that the 912- and 811-nm lines are $169 \mathrm{meV}$ and $2 \times 169 \mathrm{meV}$ away from the ZPL, respectively. Consequently, we believe the 811- and 912-nm lines are due to a $169.28(4)-\mathrm{meV}$ phonon mode and that the other lines are due to a distribution of phonon modes. Figure 3(b) shows the ${ }^{3} E \rightarrow{ }^{3} A_{2}$ fluorescence spectrum taken at $4 \mathrm{~K}$ with a similar diamond (also $\sim 10 \mathrm{ppm} \mathrm{NV}{ }^{-}$concentration). This PSB has a broader energy range, and has features at 686, 692, and $696 \mathrm{~nm}$. Using these measured spectra and the techniques outlined in Refs. 28, 35, and 36, we calculated the ${ }^{1} E \rightarrow{ }^{1} A_{1}$ and ${ }^{3} E \rightarrow{ }^{3} A_{2}$ Huang-Rhys parameters ( 0.9 and 3.49, respectively) as well as their one-phonon spectra (Fig. 4), which are the rates at which these transitions create one phonon of a given energy. We expect these one-phonon spectra to be comparable, since both come from $E \rightarrow A$ transitions with similar final-state electronic configurations [Fig. 1(c)]. The one-phonon spectra show resemblance, and the differences between them are because of electronic Coulomb repulsion corrections to the ${ }^{1} A_{1}$ level. These corrections mix the ${ }^{1} A_{1}$ level with the higher-energy ${ }^{1} A_{1}^{\prime}$ level. As a result, the ${ }^{1} A_{1}$ level contains an admixture of configurations, which results in the difference in the one-phonon spectra. ${ }^{35}$

We observed the above ${ }^{1} E \rightarrow{ }^{1} A_{1}$ PSB features in several diamond samples, and the absorption was greater in samples with higher $\mathrm{NV}^{-}$concentration. The ${ }^{1} E \rightarrow{ }^{1} A_{1}$ absorption should increase with pump power and saturate when the pumping rate becomes comparable to the ${ }^{1} E$ decay rate. The absorption at room temperature increased linearly with pump power (up to $60 \mathrm{~mW}$ focused to a minimum beam waist smaller than $5 \mu \mathrm{m}$ ), indicating that the ${ }^{1} E$ population was not saturated. However, the absorption at $10 \mathrm{~K}$ saturated at $\sim 15 \mathrm{~mW}$. This saturation is likely due to the prolonged ${ }^{1} E$ lifetime 

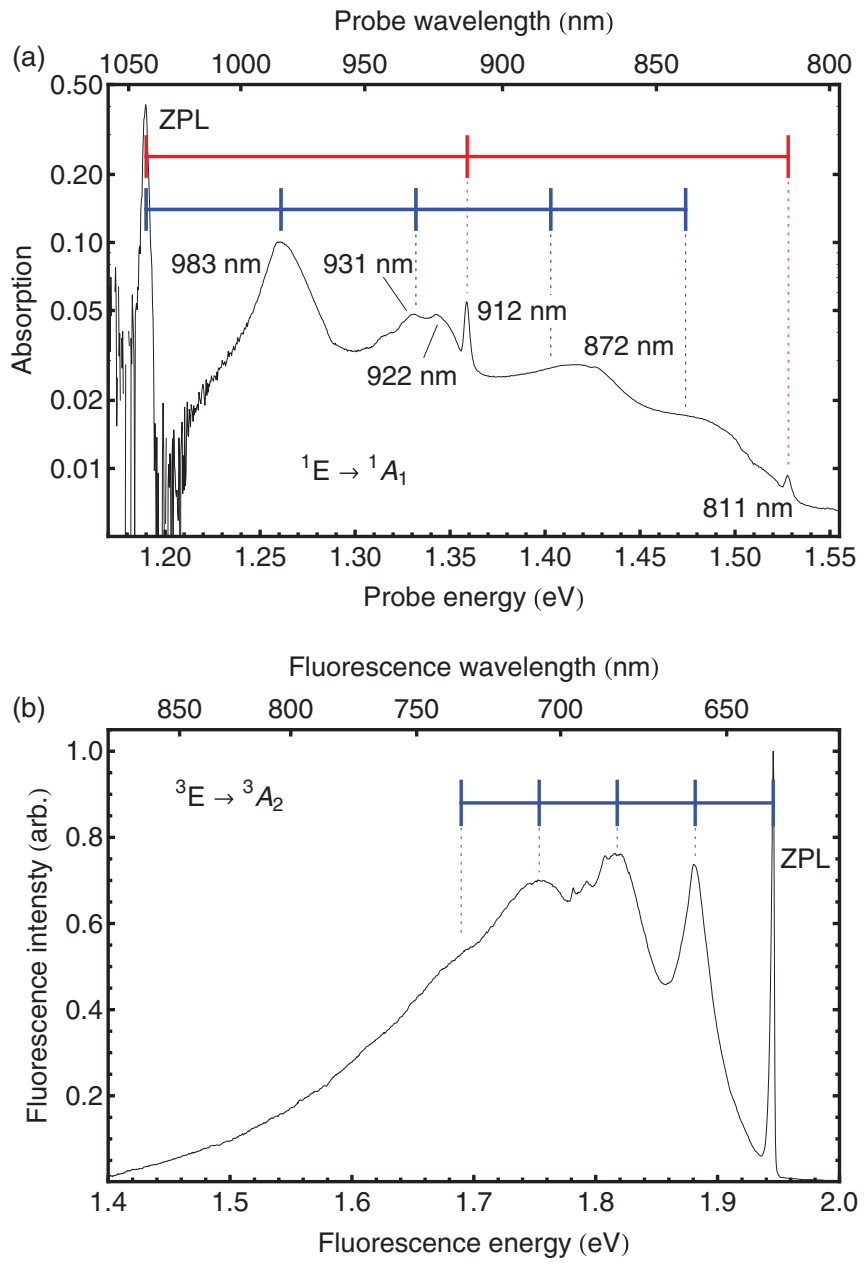

FIG. 3. (Color online) (a) The supercontiuum absorption spectrum collected at $10 \mathrm{~K}$ for diamond sample B8 using $35 \mathrm{~mW}$ of pump-laser light. PSB fluorescence from ${ }^{3} E \rightarrow{ }^{3} A_{2}$ is present for wavelengths shorter than $840 \mathrm{~nm}$ and has been subtracted out. The vertical ticks indicate the expected PSB absorption energies for 71and $169-\mathrm{meV}$ phonons, which align with some of the absorption features. (b) The fluorescence spectrum of a similar diamond collected at $4 \mathrm{~K}$. The vertical ticks indicate the expected PSB absorption energies for 64-meV phonons. Although the 686-, 692-, and 696-nm features are often ignored, they are vital to our comparison of the ${ }^{1} E \rightarrow{ }^{1} A_{1}$ and ${ }^{3} E \rightarrow{ }^{3} A_{2}$ PSBs, as they give rise to peaks (3)-(5) in Fig. 4.

at cryogenic temperature. ${ }^{18}$ Introducing a static transverse magnetic field to the samples improved the absorption contrast by a few percent. This is because the Zeeman interaction mixes the triplet spin sublevels, which spoils the optical pumping to $m_{s}=0$ and increases the ${ }^{1} E$ population.

We did not detect a ${ }^{1} E \rightarrow{ }^{1} E^{\prime}$ ZPL in the 480-1100-nm range of the supercontinuum transmission spectrum, which means this transition lies outside of this range or was too weak to detect. This wavelength span was limited by the spectrometer.

Using a rate equation calculation based on the $\mathrm{NV}^{-}$excitation and decay rates at room temperature, ${ }^{34}$ we estimate the room temperature ${ }^{1} E \rightarrow{ }^{1} A_{1}$ ZPL cross section to be roughly $4 \times 10^{-22} \mathrm{~m}^{2}$, which is consistent with previous work. ${ }^{35,37}$ The accuracy of this cross-section estimate is primarily limited

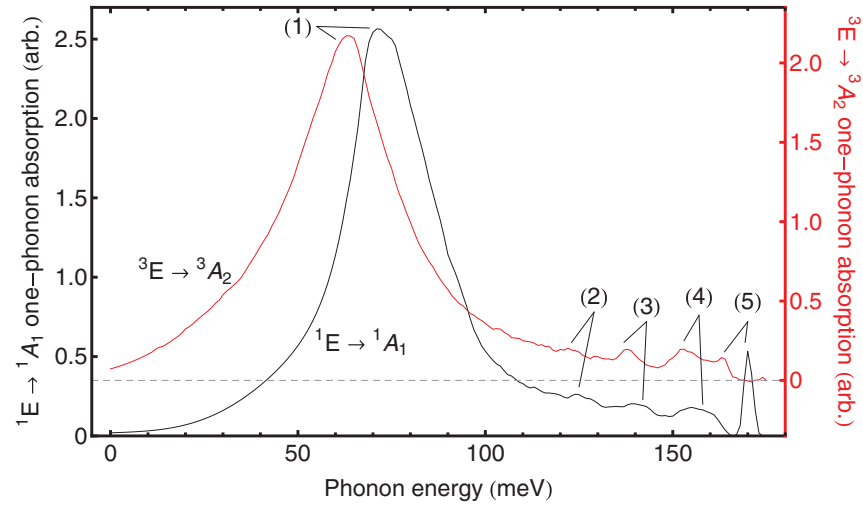

FIG. 4. (Color online) The one-phonon spectra for the ${ }^{1} E \rightarrow{ }^{1} A_{1}$ and ${ }^{3} E \rightarrow{ }^{3} A_{2}$ transitions, extracted from Fig. 3. The above spectra are normalized to have equal areas, and the ${ }^{3} A_{2}$ curve is vertically offset for clarity. In each spectrum we see five peaks, labeled (1)-(5), though the ${ }^{1} A_{1}$ peaks are shifted to higher energies (see Table I).

by uncertainty in the $\mathrm{NV}^{-}$concentration; varying the $\mathrm{NV}^{-}$ concentration from 5 to $20 \mathrm{ppm}$ in our model yields estimated cross sections ranging from 3.4 to $5.4 \times 10^{-22} \mathrm{~m}^{2}$ (compared to $4.0 \times 10^{-22} \mathrm{~m}^{2}$ with $10 \mathrm{ppm} \mathrm{NV}^{-}$).

We varied the temperature of sample B8 from 10 to $300 \mathrm{~K}$ and recorded the absorption-feature contrasts, linewidths, and integrated areas. ${ }^{35}$ The features become weaker and broader with increasing temperature, and their integrated areas decrease. This decrease in area is consistent with the ${ }^{1} E$ lifetime decrease observed in Ref. 18.

Using cw probe lasers and a similar diamond sample "S2" (16 ppm NV- concentration), we measured the center wavelengths of the 912- and 1042-nm absorption lines at $40 \mathrm{~K}$ to be 912.19(2) and 1041.96(2) nm. Our ZPL center wavelength is consistent with previous measurements. ${ }^{18,19}$ At low temperatures, the 1042- and 912-nm features have narrow widths (currently limited by the spectrometer resolution). These narrow widths imply that the vibrational mode associated with the 912-nm feature is sharp. By measuring 912-nm absorption as a function of light polarization angle, we found that the 912-nm absorption has the same polarization selection rules as the ${ }^{1} E \rightarrow{ }^{1} A_{1}$ ZPL. ${ }^{18,35}$

A 532-nm pump laser may excite other defects besides $\mathrm{NV}^{-}$ ( such as $\mathrm{NV}^{0}$ ), meaning we must be cautious when associating the observed infrared absorption features with the $\mathrm{NV}^{-1} E \rightarrow$ ${ }^{1} A_{1}$ transition. Selective excitation of infrared fluorescence using 637-nm pump light was shown in Ref. 20, meaning that while the 1042-nm ZPL is surely related to $\mathrm{NV}^{-}$, we must convince ourselves that the other infrared absorption features are also part of this electronic transition. The one-phonon absorption spectrum (Fig. 4), the optically detected magnetic resonance test of the $912-\mathrm{nm}$ selection rules, ${ }^{35}$ and the fact that a transverse magnetic field enhances the infrared absorption all confirm that our absorption spectrum belongs to the $\mathrm{NV}^{-}$ ${ }^{1} E \rightarrow{ }^{1} A_{1}$ transition.

\section{ANALYSIS AND DISCUSSION}

Comparing the ${ }^{1} E \rightarrow{ }^{1} A_{1}$ absorption PSB in Fig. 3(a) with previous observations of the ${ }^{1} A_{1} \rightarrow{ }^{1} E$ fluorescence PSB, ${ }^{19,20}$ it is evident that these PSBs differ significantly. This difference 
TABLE I. The energies of the one-phonon peaks shown in Fig. 4. When comparing the energies of the ${ }^{3} A_{2}$ and ${ }^{1} A_{1}$ phonon modes, we see a systematic shift to higher energy of a few $\mathrm{meV}$.

\begin{tabular}{lrr}
\hline \hline Peak \# & ${ }^{3} A_{2}$ state & ${ }^{1} A_{1}$ state \\
\hline$(1)$ & $64 \mathrm{meV}$ & $71 \mathrm{meV}$ \\
$(2)$ & $122 \mathrm{meV}$ & $125 \mathrm{meV}$ \\
$(3)$ & $138 \mathrm{meV}$ & $141 \mathrm{meV}$ \\
$(4)$ & $153 \mathrm{meV}$ & $156 \mathrm{meV}$ \\
$(5)$ & $163 \mathrm{meV}$ & $169 \mathrm{meV}$ \\
\hline \hline
\end{tabular}

is due to the anharmonicity of the ${ }^{1} E$ vibronic levels induced by the dynamic Jahn-Teller effect, which is not present in ${ }^{1} A_{1} \cdot{ }^{20}$ In the low-temperature limit, the PSB features of $A \rightarrow E$ electronic transitions exhibit anharmonicity, while the PSB features of $E \rightarrow A$ transitions are harmonic. ${ }^{38}$ Consequently, it is appropriate to compare the ${ }^{1} E \rightarrow{ }^{1} A_{1}$ absorption PSB with the ${ }^{3} E \rightarrow{ }^{3} A_{2}$ fluorescence PSB. Furthermore, ${ }^{1} A_{1}$ and ${ }^{3} A_{2}$ have the same electronic configuration $\left(a_{1}^{2} e^{2}\right)$ when electronic Coulomb repulsion is ignored, meaning they should have similar nuclear equilibrium positions and phonon modes. Since their initial states are different, the ${ }^{1} E \rightarrow{ }^{1} A_{1}$ and ${ }^{3} E \rightarrow{ }^{3} A_{2}$ transitions may couple to a different number of phonon modes and have different Huang-Rhys parameters, but the ${ }^{1} A_{1}$ and ${ }^{3} A_{2}$ one-phonon spectra should be similar.

As mentioned above, we extracted the one-phonon spectra from the PSBs shown in Fig. 3. The $n$-phonon spectrum is the convolution of the (n-1)-phonon and one-phonon spectra, and the sum of all $n$-phonon spectra generates the transition PSB. The one-phonon spectra are also related to the ${ }^{1} A_{1}$ and ${ }^{3} A_{2}$ phonon density of states (DOS). As seen in Fig. 4, we found similarities between the one-phonon spectra; both spectra have one large feature and four small features. However, all of the ${ }^{1} E \rightarrow{ }^{1} A_{1}$ features are displaced to higher energies (see Table I).

Introducing a point defect into a lattice alters the vibrational motion of the defect and its neighbors from what it would have been with ordinary atoms in the lattice. This is because the parameters that determine the frequencies of the vibrational motion for these atoms (the masses and effective spring constants) are modified. When the frequencies of the local oscillations of the defect lie within the spectrum of allowed vibrational modes of the remaining crystal, the local modes hybridize with the lattice modes and are called "quasilocal" (quasilocal because the nuclear oscillation amplitudes fall off slowly with increasing distance from the defect)..$^{30,36}$ The 71-meV phonon modes we observed appear to be from a quasilocal mode of $\mathrm{NV}^{-}$in the ${ }^{1} A_{1}$ state. The diamond lattice phonon DOS is appreciable at $71 \mathrm{meV},{ }^{39,40}$ and since the $\mathrm{NV}^{-}$ 71-meV mode couples strongly to the diamond lattice modes, the peaks of the 71-meV mode are consequently broadened.

In contrast to the quasilocal mode case, a "localized" mode occurs when the frequency of the local oscillations of a defect lies outside the lattice phonon DOS. In this instance, the oscillations of the defect couple poorly to the oscillations of the rest of the crystal, the vibrational motion is confined to the region of the defect, and the local phonon mode energy is unbroadened. This is the case for the $169-\mathrm{meV}$ mode. The diamond lattice phonon DOS has an upper limit of $168 \mathrm{meV}^{39-41} \mathrm{The} \mathrm{NV}^{-} 169 \mathrm{meV}$ mode falls outside the diamond lattice phonon spectrum and couples poorly to the lattice modes, consequently making the peaks of the $169-\mathrm{meV}$ mode in Fig. 3(a) sharp.

The existence of a $169-\mathrm{meV}$ local phonon mode and the differences between the ${ }^{1} E \rightarrow{ }^{1} A_{1}$ and ${ }^{3} E \rightarrow{ }^{3} A_{2}$ one-phonon spectra are surprising for several reasons. Ab initio calculations for the $\mathrm{NV}^{-}$triplet-state vibrations do not predict the existence of high-energy local phonon modes, ${ }^{29,30}$ and the ${ }^{1} E \rightarrow{ }^{1} A_{1}$ PSB is the only $\mathrm{NV}^{-}$PSB to contain such a feature. Due to the discrepancy in one-phonon spectra, we conclude that the ${ }^{1} A_{1}$ level has electronic Coulomb repulsion corrections that modify its phonon modes from those of the ${ }^{3} A_{2}$ level. Since the features in the one-phonon spectrum are shifted to higher energies, we can determine that the nearby atoms are more tightly bonded in the ${ }^{1} A_{1}$ level than in the ${ }^{3} A_{2}$ level.

\section{OUTLOOK}

In summary, we measured the ${ }^{1} E \rightarrow{ }^{1} A_{1}$ absorption spectrum of the $\mathrm{NV}^{-}$center using pump-probe spectroscopy. In the ${ }^{1} E \rightarrow{ }^{1} A_{1}$ PSB and one-phonon absorption spectrum we found several phonon modes, one of which lies outside the diamond lattice phonon DOS. The ${ }^{1} E \rightarrow{ }^{1} A_{1}$ and ${ }^{3} E \rightarrow{ }^{3} A_{2}$ one-phonon spectra show general similarity, but the ${ }^{1} A_{1}$ phonon modes are shifted to higher energies, which is from corrections to the ${ }^{1} A_{1}$ orbital configuration due to electronic Coulomb repulsion (not included in other theories). Our measurement of the ${ }^{1} E \rightarrow{ }^{1} A_{1}$ absorption spectrum shows that the ZPL is more absorptive than the PSB, and hence the ZPL offers greater sensitivity for infrared-absorption-based magnetometry than the PSB wavelengths. Furthermore, the $\mathrm{NV}^{-}$ISC and optical pumping process can be modeled more precisely using our measured ${ }^{1} A_{1}$ vibronic structure.

We searched for the ${ }^{1} E \rightarrow{ }^{1} E^{\prime} \mathrm{ZPL}$ for energies up to $2.0 \mathrm{eV}$ at cryogenic temperature and $2.6 \mathrm{eV}$ at room temperature, but we did not detect it. The ${ }^{3} A_{2} \rightarrow{ }^{3} E$ and ${ }^{1} E \rightarrow{ }^{1} E^{\prime}$ should have similar cross sections because they are transitions from electronic configuration $a_{1}^{2} e^{2}$ to $a_{1} e^{3}$ (neglecting Coulomb coupling). Since the ${ }^{1} E \rightarrow{ }^{1} A_{1}$ ZPL cross section is smaller than that of ${ }^{3} A_{2} \rightarrow{ }^{3} E$ (see Ref. 42 ), the ${ }^{1} E \rightarrow{ }^{1} E^{\prime}$ transition should have a similar or larger cross section compared to the ${ }^{1} E \rightarrow{ }^{1} A_{1}$ transition. This means the ${ }^{1} E \rightarrow{ }^{1} E^{\prime} \mathrm{ZPL}$ would likely have been detected in our absorption measurements if its energy is less than $2.0 \mathrm{eV}$. This suggests that the ${ }^{1} E \rightarrow{ }^{1} E^{\prime}$ $\mathrm{ZPL}$ energy is greater than $2.0 \mathrm{eV}$. Followup experiments will extend the search for the ${ }^{1} E \rightarrow{ }^{1} E^{\prime}$ ZPL to higher energies with improved sensitivity.

\section{ACKNOWLEDGMENTS}

We are grateful to the group of Professor F. Wang (UC Berkeley) for help with the supercontinuum laser. We thank V. Acosta and C. Santori (Hewlett-Packard Laboratories), and V. Huxter and S. Choi (UC Berkeley) for useful discussions. This work was supported by the NSF, DOE SCGF, the AFOSR/DARPA QuASAR program, NATO SFP, IMOD, ARC (DP120102232), and the Danish Council for Independent Research in Natural Sciences. 
*pkehayias@berkeley.edu

†budker@berkeley.edu

${ }^{1}$ G. Balasubramanian, P. Neumann, D. Twitchen, M. Markham,

R. Kolesov, N. Mizuochi, J. Isoya, J. Achard, J. Beck, J. Tissler,

V. Jacques, P. R. Hemmer, F. Jelezko, and J. Wrachtrup, Nat. Mater.

8, 383 (2009).

${ }^{2}$ L. M. Pham, N. Bar-Gill, C. Belthangady, D. Le Sage, P. Cappellaro, M. D. Lukin, A. Yacoby, and R. L. Walsworth, Phys. Rev. B 86, 045214 (2012).

${ }^{3}$ J. M. Taylor, P. Cappellaro, L. Childress, L. Jiang, D. Budker, P. R. Hemmer, A. Yacoby, R. Walsworth, and M. D. Lukin, Nat. Phys. 4, 810 (2008).

${ }^{4}$ J. R. Maze, P. L. Stanwix, J. S. Hodges, S. Hong, J. M. Taylor, P. Cappellaro, L. Jiang, M. V. Gurudev Dutt, E. Togan, A. S. Zibrov, A. Yacoby, R. L. Walsworth, and M. D. Lukin, Nature (London) 455, 644 (2008).

${ }^{5}$ G. Balasubramanian, I. Y. Chan, R. Kolesov, M. Al-Hmoud, J. Tisler, C. Shin, C. Kim, A. Wojcik, P. R. Hemmer, A. Krueger, T. Hanke, A. Leitenstorfer, R. Bratschitsch, F. Jelezko, and J. Wrachtrup, Nature (London) 455, 648 (2008).

${ }^{6}$ F. Dolde, H. Fedder, M. W. Doherty, T. Nöbauer, F. Rempp, G. Balasubramanian, T. Wolf, F. Reinhard, L. C. L. Hollenberg, F. Jelezko, and J. Wrachtrup, Nat. Phys. 7, 459 (2011).

${ }^{7}$ D. Maclaurin, M. W. Doherty, L. C. L. Hollenberg, and A. M. Martin, Phys. Rev. Lett. 108, 240403 (2012).

${ }^{8}$ M. P. Ledbetter, K. Jensen, R. Fischer, A. Jarmola, and D. Budker, Phys. Rev. A 86, 052116 (2012).

${ }^{9}$ A. Ajoy and P. Cappellaro, Phys. Rev. A 86, 062104 (2012).

${ }^{10}$ J. Wrachtrup and F. Jelezko, J. Phys.: Condens. Matter 18, S807 (2006).

${ }^{11}$ P. C. Maurer, G. Kucsko, C. Latta, L. Jiang, N. Y. Yao, S. D. Bennett, F. Pastawski, D. Hunger, N. Chisholm, M. Markham, D. J. Twitchen, J. I. Cirac, and M. D. Lukin, Science 336, 1283 (2012).

${ }^{12}$ R. Alléaume, F. Treussart, G. Messin, Y. Dumeige, J.-F. Roch, A. Beveratos, R. Brouri-Tualle, J.-P. Poizat, and P. Grangier, New J. Phys. 6, 92 (2004).

${ }^{13}$ A. Beveratos, R. Brouri, T. Gacoin, A. Villing, J.-P. Poizat, and P. Grangier, Phys. Rev. Lett. 89, 187901 (2002).

${ }^{14}$ E. Rittweger, K. Y. Han, S. E. Irvine, C. Eggeling, and S. W. Hell, Nat. Photon. 3, 144 (2009).

${ }^{15}$ S. W. Hell, R. Schmidt, and A. Egner, Nat. Photon. 3, 381 (2009).

${ }^{16}$ P. C. Maurer, J. R. Maze, P. L. Stanwix, L. Jiang, A. V. Gorshkov, A. A. Zibrov, B. Harke, J. S. Hodges, A. S. Zibrov, A. Yacoby, D. Twitchen, S. W. Hell, R. L. Walsworth, and M. D. Lukin, Nat. Phys. 6, 912 (2010).

${ }^{17}$ G. Davies and M. F. Hamer, Proc. R. Soc. Lond. A 348, 285 (1976).
${ }^{18}$ V. M. Acosta, A. Jarmola, E. Bauch, and D. Budker, Phys. Rev. B 82, 201202 (2010).

${ }^{19}$ L. J. Rogers, S. Armstrong, M. J. Sellars, and N. B. Manson, New J. Phys. 10, 103024 (2008).

${ }^{20}$ N. Manson, L. Rogers, M. Doherty, and L. Hollenberg, arXiv:1011.2840.

${ }^{21}$ N. Aslam, G. Waldherr, P. Neumann, F. Jelezko, and J. Wrachtrup, New J. Phys. 15, 013064 (2013).

${ }^{22}$ D. M. Toyli, D. J. Christle, A. Alkauskas, B. B. Buckley, C. G. Van de Walle, and D. D. Awschalom, Phys. Rev. X 2, 031001 (2012).

${ }^{23}$ A. Lenef and S. C. Rand, Phys. Rev. B 53, 13441 (1996).

${ }^{24}$ P. Delaney, J. C. Greer, and J. A. Larsson, Nano Lett. 10, 610 (2010).

${ }^{25}$ M. W. Doherty, N. B. Manson, P. Delaney, and L. C. L. Hollenberg, New J. Phys. 13, 025019 (2011).

${ }^{26}$ J. R. Maze, A. Gali, E. Togan, Y. Chu, A. Trifonov, E. Kaxiras, and M. D. Lukin, New J. Phys. 13, 025025 (2011).

${ }^{27}$ S. K. Choi, M. Jain, and S. G. Louie, Phys. Rev. B 86, 041202 (2012).

${ }^{28}$ G. Davies, J. Phys. C 7, 3797 (1974).

${ }^{29}$ A. Gali, T. Simon, and J. E. Lowther, New J. Phys. 13, 025016 (2011).

${ }^{30}$ J. Zhang, C.-Z. Wang, Z. Z. Zhu, and V. V. Dobrovitski, Phys. Rev. B 84, 035211 (2011).

${ }^{31}$ B. T. Webber, M. C. Per, D. W. Drumm, L. C. L. Hollenberg, and S. P. Russo, Phys. Rev. B 85, 014102 (2012).

${ }^{32}$ V. M. Acosta, E. Bauch, A. Jarmola, L. J. Zipp, M. P. Ledbetter, and D. Budker, Appl. Phys. Lett. 97, 174104 (2010).

${ }^{33}$ L. Robledo, H. Bernien, T. van der Sar, and R. Hanson, New J. Phys. 13, 025013 (2011).

${ }^{34}$ J.-P. Tetienne, L. Rondin, P. Spinicelli, M. Chipaux, T. Debuisschert, J.-F. Roch, and V. Jacques, New J. Phys. 14, 103033 (2012).

${ }^{35}$ See Supplemental Material at http://link.aps.org/supplemental/ 10.1103/PhysRevB.88.165202 for additional experimental and theoretical details.

${ }^{36}$ A. A. Maradudin, Solid State Physics, edited by F. Seitz and D. Turnbull (Academic Press, New York, 1966), Vol. 18, p. 273.

${ }^{37}$ Y. Dumeige, M. Chipaux, V. Jacques, F. Treussart, J.-F. Roch, T. Debuisschert, V. M. Acosta, A. Jarmola, K. Jensen, P. Kehayias, and D. Budker, Phys. Rev. B 87, 155202 (2013).

${ }^{38}$ H. C. Longuet-Higgins, U. Öpik, M. H. L. Pryce, and R. A. Sack, Proc. R. Soc. London, Ser. A 244, 1 (1958).

${ }^{39}$ N. Mounet and N. Marzari, Phys. Rev. B 71, 205214 (2005).

${ }^{40}$ A. Bosak and M. Krisch, Phys. Rev. B 72, 224305 (2005).

${ }^{41}$ A. M. Zaitsev, Optical Properties of Diamond: A Data Handbook (Springer, New York, 2001).

${ }^{42}$ T.-L. Wee, Y.-K. Tzeng, C.-C. Han, H.-C. Chang, W. Fann, J.-H. Hsu, K.-M. Chen, and Y.-C. Yu, J. Phys. Chem. A 111, 9379 (2007). 